\title{
Simulation and Modelling of Pipeline Corrosion and Integrity Management in Oil and Gas Industry
}

\author{
Nse Peter Essang \\ Department of Petroleum and Natural Gas Processing Engineering, \\ Petroleum Training Institute, Effurun, Nigeria
}

\begin{abstract}
In this research work, Monte Carlo Simulation and degradation models were used to predict the corrosion rate and reliability of crude oil pipelines. Discrete random numbers simulated from Inline Inspection Data were used to predict the corrosion rate using Linear and Power Law Model. The mean time for failure (MTFF) was estimated with the degradation models. The result of the study shows that the degradation models and Monte Carlo simulation can predict the corrosion rate of the pipelines to an accuracy of between 83.05-98.33\% and 84.24-97.94\% respectively. From the plot the lowest degradation recorded was $1.67 \%$ (Power law) and highest 16.95 (Power Law), for Linear Model Law, the lowest value recorded was 2.11\% while the highest is $15.23 \%$. In comparison to the value obtained from Monte Carlo Simulation (2.01 lowest and 15.76 highest), all the values fall between $1.67 \%$ to $16.95 \%$. Thus, RMSE of between $1.67 \%$ and $16.95 \%$ was recorded for the degradation models. Therefore, the statistical models give the expected number of failures. The results of the statistical models can be used in reliability analysis, risk analysis, and optimum maintenance decisions.
\end{abstract}

\section{KEYWORDS: Corrosion rate, Simulation, Modelling, Reliability, Pipeline integrity}

\section{INTRODUCTION}

Corrosion depletes the integrity of a pipeline thereby causing it to fail before its design service life. The corrosion of pipelines can be described as a systematic degradation of the pipeline wall due to the actions of operating parameters on the pipeline material (Klechka, 2002). For effective monitoring of pipeline reliability and life prediction, corrosion risk assessment is necessary (Amirat et al., 2006). In order to manage corrosion risks, monitoring and inspection program are also required. The probability of failure is estimated based on the type of corrosion damage expected to occur while the consequences of failure are measured against the impact of such a failure evaluated against a number of criteria (Gopika et al., 2003). To prioritize inspection according to the permissible risk level involves the understanding of the consequences of failure of a component on a system This requires the analysis of the system according to stipulated standards in order to predict the remaining life. The criteria could include potential hazards to environment, risks associated with safety and integrity, or risk due to corrosion or inadequate corrosion mitigation procedure (Ahmad et al., 2011). Pipeline used in oil and gas production fail due to factors that are operationally, structurally and environmentally induced. The operational factors are associated with the components of the fluid flowing through while the environmental factors deal with the electrochemical and mechanical interactions of the pipeline material and the immediate surroundings (Alfon et al., 2012).

Pipeline Integrity Management (PIM) is the cradle-to-grave approach of understanding and operating pipelines in a safe, reliable manner (ASME, 2010). Pipeline infrastructures represent a high capital investment and pipelines must be free from the risk of degradation which could cause environmental hazards and potential threats to life, pipeline integrity design, monitoring and management become very crucial. Pipelines are the most popular method for transporting crude oil and natural gas. Pipelines distribute almost 70\% of oil and gas products worldwide (Mohitpour et al., 2010). Also, pipeline networks are growing every year due to pipelines construction. Pipelines require the highest level of reliability due to safety concerns. In fact, pipeline systems are becoming more complex and being located excessively near high-density populated area. Pipeline failure due to corrosion of oil and gas facilities can occur in between planned inspection intervals. Considering the risk involved in pipeline failure which could results from corrosion, it became necessary to develop a model for the operators to estimate the number of future failures. With such model, the operators can minimize the operational risks such as injury, fatality, economical, and etc. Also, the operators can mitigate and control most pipeline failures. They can produce safer operations, and they can decide on effective maintenance procedures and timing.

\section{Materials and Methods}

The effect of change in temperature of pipeline restrained in axial direction generally induces axial stresses by the restrained thermal strains of:

$$
\varepsilon=\alpha \Delta \sigma
$$

For the prediction of corrosion wastage of the pipeline, annual corrosion rate (ACR) was used to give the best estimate of the pipeline corrosion rate. This was achieved by comparing the predicted corrosion rates with the field data using root mean square error (RMSE). The RMSE can be calculated from Equation (2);

$$
\text { RMSE }=\sqrt{\frac{1}{n} \sum_{i=1}^{n}\left(C R_{p}-C R_{i}\right)^{2}}
$$

$\mathrm{n}=$ number of years of used corrosion data $\mathrm{CR}_{\mathrm{p}}=$ Predicted Corrosion rate for ith year $\mathrm{CR}_{\mathrm{i}}=$ Measured field corrosion rate for ith year from Inlineinspection data 
In this research work, the ACR with the lower RMSE is used to predict the corrosion wastage of the pipelines for the year 2000, 2005 and 2010 respectively. The corrosion wastage represents the cumulative wall thickness loss of the pipeline at any time interval. The understanding of corrosion wastage helps in decisions concerning risks based inspection (RBI). To predicted corrosion rates, the best ACR (Equation 3 ) for the period of the years; $1,2, \ldots$, i. is $C_{1}, C R_{2}, \ldots, C R i$, are used for the corrosion wastage for the nth year $\left(\mathrm{CR}_{n}\right)$ given by Equation (4);

$$
\begin{aligned}
& \frac{\delta_{C R}}{C R_{p}}=e^{\mu \delta T+\sigma s(\delta T)^{\frac{1}{2}}} \\
& C R_{n}=C R_{1}+C R_{2}+\ldots+C R_{i}=\sum_{i=1}^{n} C R_{i}
\end{aligned}
$$

where,

$\delta_{\mathrm{CR}}=$ Change in the corrosion rate from one year to year $\mathrm{CR}_{\mathrm{p}}=$ Previous value of the corrosion rate $\mu=$ average value of the corrosion rate in each pipeline $\sigma=$ Annualized volatility or standard deviation of the corrosion rate

$\delta \mathrm{T}=$ change in time (in years) from one step to another $s=$ Probability distribution

Degradation is a process of loss of integrity and function of a system due to ageing, operation and other factors which could include environmental and human factors. To predict the remaining life of the pipeline requires the estimation of the rate of degradation, reliability and the remaining life. Degradation is a continuous progression of wear and decay, so it can be modeled as a stochastic process. The measured degradation for $i_{\text {th }}$ tested device $(i=1,2, . . n)$ will consist of a vector of $m_{i}$ measurements made at time points $\mathrm{ti}_{1}, \ldots \mathrm{ti}_{\mathrm{mi}}$. At the $m_{i}$ time point, the degradation measurement $\left(Y_{i j}\right)$ of device $\mathrm{i}$ is given by Equation (5).

$$
Y_{i j}=\mathrm{n}\left(t_{i j}\right)+\varepsilon_{i j}, 1<i \leq n, i \leq j \leq m_{i}
$$

where,

tij = Pipe wall thickness

The form of degradation ( $\eta$ ) can be chosen to have a strict form or it can be more arbitrary. The forms of $\eta$ used to determine the degradation of the pipeline is shown in linear model (Equations 6) and power law model (Equation 7).

$$
\begin{aligned}
& \mathrm{CR}=\alpha \mathrm{T}_{\mathrm{m}}+\beta \\
& \mathrm{CR}=\beta\left(\mathrm{T}_{\mathrm{m}}\right)^{\mathrm{a}}
\end{aligned}
$$

where,

$\mathrm{CR}=$ degradation of pipeline due to corrosion

$\mathrm{T}_{\mathrm{m}}=$ Time

$\alpha$ and $\beta=$ Constants of model parameters

The above degradation model equations were used to estimate the time of failure of the pipeline. The time for failure is assumed to be reached when the corroded wall thickness is $45 \%-85 \%$ of the original wall thickness. The commuted time of failure (mean time for failure) was used for the life data analysis. The mean time for failure (MTFF) for the pipeline was established using the degradation model according to the relationship shown in Equation (8).

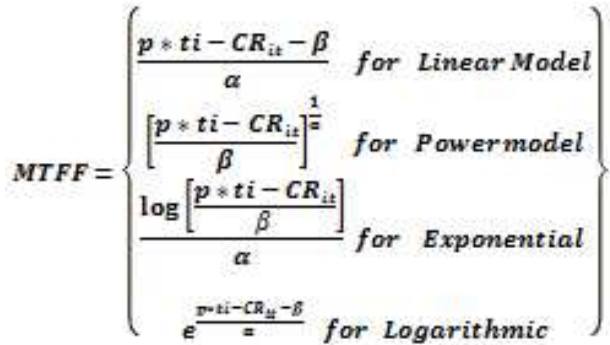

$\mathrm{P}=$ percentage of corroded wall thickness (45\%-85\%) $\mathrm{CR}_{\mathrm{it}}=$ Measured corrosion rates along the pipeline at years $(1,2, \ldots, n)$

The pipe is manufactured seamless operating three phase crude in OML 23, Niger Delta Nigeria. The pipeline was commissioned in 1995, is coal-tar coated. The property of the pipeline is shown in Table (1) while pipeline data of 2000, 2005, and 2010 were used. The establishment of thickness measurement location (TML) area along the length of the pipeline made matching the corrosion defects from both inspections under the limit that, in addition to having the same location, their depths in the second and third inspection must be equal to or larger than that of the first and second inspection respectively, preceded use of the ILI data. The matching process considered typical odometer and depth measurement errors of the MFL-ILI tools. Only the matched defects were part of the analyses that followed, ensuring that only actual defect progression with time was considered without including defects that might have occurred in the interval between the three inspections. ILI data are named "2000-ILI", "2005-ILI", and "2010-ILI" referring to the matched defect populations measured in 2000, 2005 and in 2010, respectively. Depth distribution of the defects in 2000-ILI were fed into each one of the CR growth models under assessment as the initial depth distribution. Comparing this distribution to that of defects observed in 2005-ILI best describes the observed pit-depth evolution over the 5-year interval between the two. Similar assessment was also carried for the second ILI data. Data from both inspections based on the observed change in depth of the matched defects over the interval, $\delta$ t, helped determine an empirical CR distribution, which was then compared with the corrosion rate distributions predicted by the CR models under assessment.

Table 1: Pipeline Data

\begin{tabular}{|c|c|}
\hline Pipe Material & API 5L G X-56 Steel \\
\hline Yield Strength & $56 \mathrm{KSI}$ \\
\hline Ultimate Tensile Strength & $71 \mathrm{KSI}$ \\
\hline Length of Pipeline & $16 \mathrm{Km}$ \\
\hline Pipe Outer Diameter (OD) & $323.90 \mathrm{~mm}$ \\
\hline Specified Wall Thickness & $12.7 \mathrm{~mm}$ \\
\hline Design Pressure & $80.90 \mathrm{Bar}$ \\
\hline Operating Pressure & $56.63 \mathrm{Bar}$ \\
\hline Design Temperature & $100^{\circ} \mathrm{C}$ \\
\hline Operating Temperature & $40^{\circ} \mathrm{C}$ \\
\hline
\end{tabular}

\section{Results and Discussion}

Degradation analysis was performed on Inline-inspection data using linear and power models. Microsoft Excel analysis tool was used for the regression analysis and determination of the model constants that were used for predicting the best fit equations. These predicted equations of best fit were used for estimating the MTFF at each of the 
measured corrosion rate in the field by applying Equation (8). In this research work, the value of $\mathrm{p}$ was assumed to be $45 \%$ while the wall thickness of the pipeline is $10 \mathrm{~mm}$. Thickness measurement location was carried out and the outcome confirm the presence of corrosion between the year 2000, 2005 and 2010 respectively (Figure 1). The values obtained for the year 2000, 2005 and 2010 were used to determine the mean and standard deviation of Inlineinspection data corrosion rate ( $\mathrm{mm} /$ year). There was an increase in mean rate of corrosion between the year of study (2000, 2005 and 2010). The mean corrosion rates, standard deviation and most frequent portion of corrosion of the simulation runs are used as the annualized corrosion rate
(ACR) for the so purpose of predicting the yearly corrosion rate and corrosion wastage. The ACR that has the least error is used for the prediction of the pipeline corrosion rate for the future. Figure 2 shows the plot of TML against Root Mean Square Error (RMSE) of Predicted Models. From the plot the lowest degradation recorded was $1.67 \%$ (Power law) and highest 16.95 (Power Law), for Linear Model Law, the lowest value recorded was $2.11 \%$ while the highest is $15.23 \%$. In comparison to the value obtained from Monte Carlo Simulation (2.01 lowest and 15.76 highest), all the values fall between $1.67 \%$ and $16.95 \%$. Therefore, RMSE of between $1.67 \%$ and $16.95 \%$ was recorded for the degradation models.
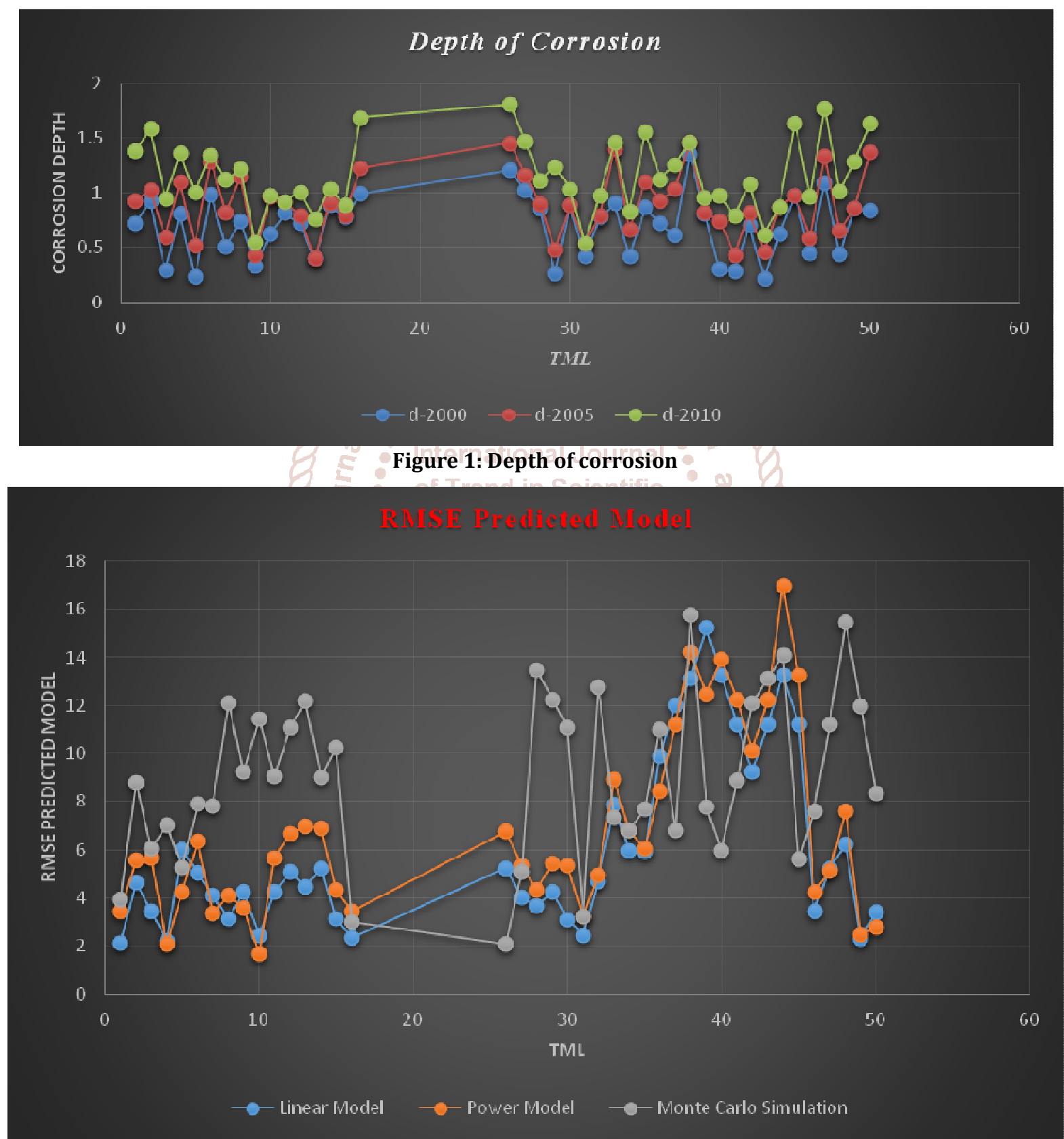

Figure 2: RMSE Predicted Model

Moreover, the average corrosion rate (CRav) obtained from Monte Carlo simulation was able to predict the pipeline corrosion rate to an accuracy of between $84.24 \%-97.94 \%$ (Table 4.2 ). Thus, using it for future pipeline corrosion prediction will give the experts a good idea of the reliability of the pipelines for enhanced integrity management. Figure 3 shows the variation of Inlineinspection data (field data) measured and predicted (Monte Carlo simulation (CRpred) and Degradation Model) corrosion rates for the pipelines. The value of $\mathrm{R}^{2}$ ranges between 0.806- 0.991 for the Linear Model and 0.807-0.995 for the Power Model. The outcome of the results shows that the degradation model has predicted the field corrosion of the pipelines to a high degree of accuracy and hence will be a vital tool for predicting the failure time of the pipelines. 


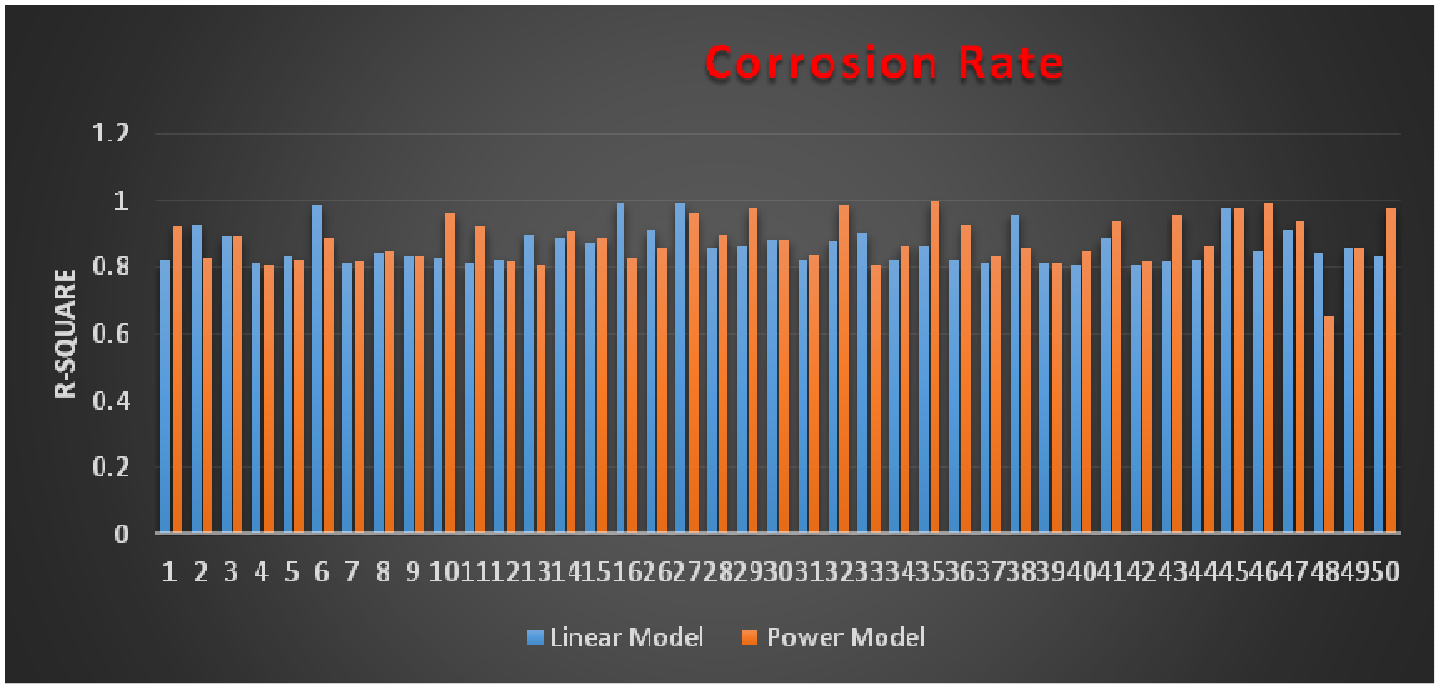

Figure 3: Corrosion Rates for Pipelines

\section{Conclusion}

This research work is aims to establish pipeline failure and corrosion rate prediction using a reliability model for pipeline integrity and safety. In this research work, Monte Carlo simulation and Degradation Models were used to predict pipeline corrosion and establish the reliability of the pipeline over a certain period. Inline Inspection data (Field data) was used for this analysis. The corrosion prediction involved the use of Linear and Power models and estimation of mean time for failure (MTFF) of the pipelines. The statistical models give the expected number of failures. The results of the statistical models can be used in reliability analysis, risk analysis, and optimum maintenance decisions. The outcome of the research work showed that both the Monte Carlo Simulation and the Degradation Models can give better prediction of the pipeline corrosion rate.

\section{References}

[1] Ahmad, I., Gazwi, R. H., and Elosheby, I. I. (2011). Pipeline Integrity Management Through Corrosion Mitigation and Inspection Strategy in Corrosive Environment: An Experience of Arabian Gulf Oil Company in Libya. Proceeding of NACE - International Corrosion Conference Series, Houston, TX.
[2] Alfon, P., Soedarsono, J. W., Priadi, D., and Sulistijono, S. (2012). Pipeline Material Reliability Analysis Regarding to Probability of Failure Using Corrosion Degradation Model. Advanced Materials Research, 422, pp.705-715.

[3] American Society of Mechanical Engineers (ASME). (2010). Managing System Integrity of Gas Pipelines (ASME B31.8S-2010), New York, NY

[4] Amirat, A., Mohamed-Chateauneuf, A. and Chaoui, K. (2006): Reliability assessment of underground pipelines under the combined effect of active corrosion and residual stress. International Journal of Pressure Vessels and Piping, Vol. 83, pp. 107-117

[5] Gopika, V., S.K. Bidhar, H.S. Kushwaha, A.K. Verma, A. Srividya (2003): A comprehensive framework for evaluation of piping reliability due to erosioncorrosion for risk- informed in service inspection. Reliability Engineering \& System Safety vol. 82, Issue 2, pp.187.

[6] Klechka, E.W. (2002): Pipeline integrity management and corrosion control. Materials Performance; (2), pp.4-7 